Pacific Journal of Mathematics

COMPACTS DE ROSENTHAL 


\title{
COMPACTS DE ROSENTHAL
}

\section{GiLles Godefroy}

\begin{abstract}
We study in this work a class of compacts spaces which extends in a natural way the class of metrizable compact spaces. We clarify stability properties of this class under the usual operations, and some topological properties of these

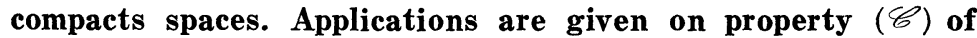
Banach spaces, on sub-algebras of $\ell^{\infty}(N)$, on Boolean subalgebras of $\mathscr{P}(N)$.
\end{abstract}

Remerciements. Je remercie vivement Michel Talagrand pour ses nombreuses et précieuses indications qui m'ont permis de mener ce travail à bien; cet article, sans son aide, n'aurait pas vu le jour.

On appelle dans ce travail "compacts de Rosenthal" les espaces compacts qui sont homéomorphes à des compacts de fonctions de première classe de Baire sur un espace métrique complet séparable $P$, la topologie considérée étant la topologie de la convergence ponctuelle sur l'espace $P$.

Les compacts de Rosenthal furent introduits par H. P. Rosenthal afin de caractériser les espaces de Banach séparables qui contiennent un sous-espace isomorphe à $\ell^{1}(N)$ (voir par example [10], [8]). Ils ont été ensuite étudiés en détail par J. Bourgain, D. H. Fremlin et M. Talagrand ([3]). Tout compact métrisable $K$ est un compact de Rosenthal; en effet $K$ apparaît comme un compact de fonctions continues sur l'espace métrique complet séparable $\mathscr{C}(K)$. Il existe des compacts de Rosenthal séparables non métrisables; citons-en quatre exemples:

- Le "compact de Helly" des fonctions croissantes de $[0,1]$ dans lui-même.

- Le compact $[0,1] \times\{0,1\}$ muni de l'ordre lexicographique ("intervalle éclaté").

- Le compact $K=\left[f:[0,1] \rightarrow R \mid f(0)=0, V_{0}^{1} f \leqq 1\right\}$

- La boule unité du bidual d'un espace de Banach séparable ne contenant pas $\ell^{1}(N)$ et à dual non séparable ([7]), munie de la topologie pré-faible $\varkappa^{*}$.

Les compacts de Rosenthal forment donc une classe de compacts qui étend naturellement la classes des compacts métrisables, et qui est distincte de la classe des compacts d'Eberlein. Nous allons maintenant étudier cette classe plus en détail.

Dans un premier paragraphe, on va donner des caractérisations topologiques des compacts de Rosenthal séparables (Remarque: il 
existe des compacts de Rosenthal non séparables, par exemple $K=$ $\left.\left\{X_{\{x \mid} \mid x \in[0,1]\right\} \cup\{0\}\right)$, et en déduire les résultats suivants:

- La classe des compacts de Rosenthal n'est pas stable par image continue.

- La classe des compacts de Rosenthal séparables est stable par image continue ouverte.

- Si $K$ est un compact de Rosenthal, alors l'espace $M_{1}^{+}(K)$ des mesures de probabilité sur $K$, muni de la topologie vague, est un compact de Rosenthal.

- Si $K$ est un compact de Rosenthal, toute mesure de Radon sur $K$ a un support séparable.

Dans un deuxième paragraphe, on donnera des caractérisations utilisant l'espace $\mathscr{C}(K)$ des fonctions continues sur $K$. On en déduira divers résultats de stabilité, ainsi que quelques applications aux sousalgèbres de $\ell^{\infty}(N)$ et aux sous-algèbres de Boole de $P(N)$.

Notations ET Definitions. On appellera espace polonais tout espace métrique complet séparable. On dira qu'un espace topologique est analytique s'il est image continue de l'espace $N^{N}$; tout espace polonais est analytique; la famille des sous-ensembles analytiques de $R$ contient la tribu des boréliens. Un espace mesurable $(E, \tau)$ sera dit souslinien s'il est isomorphe en tant qu'espace mesurable, à un sous-ensemble analytique de $R$ muni de la tribu des boréliens. Une référence standard pour ces notions est [4]. Tout espace analytique est souslinien pour sa tribu borélienne. L'ensemble $P(N)$ des parties de $N$, identifié à $\{0,1\}^{N}$, sera muni de la topologie produit, qui en fait un compact métrisable. Tout filtre sur un ensemble dénombrable $D$ pourra donc être considéré comme un sous-ensemble du compact métrisable $P(D)$. On dira alors que $F$, filtre sur $D$ est un filtre analytique si $F$ s'identifie à un sous-ensemble analytique de $P(D)$.

Enfin, si $E$ est un espace de Banach, on notera w la topologie faible sur $E$, c'est-à-dire la topologie de la convergence simple sur le dual $E^{*}$ de $E$. On notera * $^{*}$ la topologie pré-faible sur $E^{*}$, c'està-dire la topologie de la convergence simple sur $E$.

Rappelons enfin qu'une fonction définie sur un espace polonais $P$ est dite de $1^{\text {ere }}$ classe de Baire si elle est limite d'une suite de fonctions continues pour la topologie de la convergence simple; une fonction $f$ est de $1^{\text {ere }}$ classe si pour tout fermé $F$ de $R$, l'ensemble $f^{1}(F)$ est un $G \delta$ de $P$.

Un espace réqulièr séparé est dit "angélique" si:

(1) Tout ensemble relativement dénombrablement compact est relativement compact.

(2) L'adhérence d'un ensemble relativement compact est exactement l'ensemble des limites des suites de cet ensemble. 
On montre que l'espaces des fonctions de $1^{\text {ere }}$ classe de Baire sur un polonais est angélique ([3]); en particulier tout compact de Rosenthal est angélique. Donc, dans un compact de Rosenthal, tout point adhérent à une partie $X$ sera limite d'une suite d'éléments de $X$.

I. Caracterisation topologique des espaces de Rosenthal applications.

Posons tout d'abord une définition.

Definition 1. Soit $A$ un ensemble dénombrable, $\mathscr{C}$ une structure uniforme $\operatorname{sur} A$. On dira que $\mathscr{C}$ est analytique si le filtre des entourages de $\mathscr{C}$ est une partie analytique de $\mathscr{P}(A \times A)$.

Pour une définition des espaces uniformes, voir [1]. On a alors le lemme.

Lemme 2. Soit $A$ un ensemble dénombrable, $\mathscr{U}$ une structure uniforme analytique sur $A$. Alors l'ensemble $S$ des fonctions bornées de $A$ dans $R$ uniformément continues relativement $\grave{a} \mathscr{Q}$ est une partie analytique de $R^{A}$.

Demonstration. On a $S=\bigcup_{n \in N}\left(S \cap B_{n}\right)$, où $B_{n}=[-n,+n]^{A}$.

Il suffit de montrer que pour tout $n$, l'espace $\left(S \cap B_{n}\right)$ est analy tique.

Considérons le compact $K_{n}=[-n,+n]^{A} \times\{0,1\}^{A \times A}$; soit $k \in N$. On pose $F_{k}=\left\{(u, X) \in K_{n}|\forall(h, 1) \in X| u,(h)-N(1) \mid \leqq(1 / k)\right\} F_{k}$ est un fermé de $K_{n}$. On a $u \in S \cap B_{n} \Leftrightarrow \forall k, \exists X \in \mathscr{C}$ tel que $(u, X) \in F_{k}$. On On a donc $S \cap B_{n}=\bigcap_{k \in N} \Pi_{1}\left(F_{k} \cap\left([-n,+n]^{A} \times \mathscr{U}\right)\right)$ où $\Pi_{1}$ est la projection de $K_{n}$ sur $[-n,+n]^{A}$. Ceci montre que $S \cap B_{n}$ est une intersection dénombrable d'analytiques, donc un analytique.

Le lemme suivant nous sera utile.

Lemme 3. Soit $X$ un espace topologique analytique. Soit $\left(f_{n}\right)_{n \in N}$ une suite de fonctions boréliennes sur $X$. Soit $F$ un filtre analytique sur $N$ tel que $\lim _{F} f_{n}$ existe; on note $f=\lim _{F} f_{n}$. Alors $f$ est une fonction borélienne sur $X$.

Demonstration. Soit $X^{\prime}=\prod_{n \in N} f_{n}(X)$.

Les fonctions $\left(f_{n}\right)$ étant boréliennes, l'espace $X^{\prime}$ est analytique. On note $\psi$ l'application de $X$ dans $X^{\prime}$ définie par

$$
\begin{aligned}
\psi: X & \longrightarrow X^{\prime} \\
x & \longrightarrow\left(f_{n}(x)\right)_{n \in N} .
\end{aligned}
$$

On a alors $f_{n}=\Pi_{n} \circ \gamma$. On considère l'espace $E=X^{\prime}\{\times 0,1\}^{N}$. On a 
$f(x) \in[\alpha, \beta] \Leftrightarrow \forall \varepsilon, \exists \varphi \in F$ tel que $f_{n}(x) \in[\alpha-\varepsilon, \beta+\varepsilon] \forall n \in \varphi$. On pose alors $\mathscr{C}_{\varepsilon}=\left\{(u, \varphi) \in E \mid \Pi_{n}(u) \in[\alpha-\varepsilon, \beta+\varepsilon] \forall n \in \varphi\right\} \mathscr{C}_{\varepsilon}$ est un fermé de E. On a

$$
\begin{gathered}
\exists \varphi \in F \text { tel que } f_{n}(x) \in[\alpha-\varepsilon, \beta+\varepsilon] \forall n \in \varphi \\
\psi(x) \in \prod_{1}\left(\mathscr{C}_{\varepsilon} \cap\left(K^{\prime} \times F\right)\right)
\end{gathered}
$$

done

$$
f^{-1}([\alpha, \beta])=\bigcap_{n \in N} \psi^{-1}\left[\prod_{1}\left(\mathscr{C}_{1 / n} \cap\left(K^{\prime} \times F\right)\right)\right]
$$

$f^{-1}(\alpha, \beta)$ est donc analytique dans $X$ pour tout couple $(\alpha, \beta)(\alpha<\beta)$ de réels d'après l'analyticité de $F$ et le caractère borélien de $\psi$; le théorème de séparation montre alors que $f$ est borélienne.

On peut à présent énoncer le résultat principal.

Theoreme 4. Soit $K$ un compact séparable. Les énoncés suivants sont équivalents:

(1) $K$ est un compact de Rosenthal.

(2) Pour toute partie dénombrable $D$ dense $K$, la structure uniforme $\mathscr{C}_{D}$ induite sur $D$ par la structure uniforme de $K$ est analytique.

(3) Pour toute partie dénombrable $D$ dense dans $K$, l'espace $\mathscr{C}(K)$ muni de la topologie de la convergence simple sur $D$ est analytique.

Demonstration. $(1) \Rightarrow(2)$. Soit $K$ un compact de Rosenthal séparable, $D$ une partie dénombrable dans $K$. Soit $X$ espace polonais tel que $K$ soit homeomorphe à un compact de $B_{1}(X)$, espace des fonctions de première classe sur $X$. On note $D=\left\{f_{n}\right\}_{n \in N}$. Soit $\psi$ définie par

$$
\begin{aligned}
& \psi: X \times N \longrightarrow \mathscr{P}(D \times D) \\
& (x, k) \longmapsto\left\{\left(f_{n}, f_{p}\right) \in|D \times D|\left|f_{n}(x)-f_{p}(x)\right| \leqq \frac{1}{k}\right\} .
\end{aligned}
$$

Les fonctions $\left\{f_{n}\right\}$ étant boréliennes sur $X$, on vérifie aisément que $\psi$ est borélienne. Par conséquent $\psi(X \times N)$ est un sous-ensemble analytique de $\mathscr{P}(D \times D)$. Le filtre des entourages de $\mathscr{U}_{D}$ est le filtre engendré par $\psi(X \times N)$; or un filtre engendré par un sous-ensemble analytique de $\mathscr{P}(D \times D)$ est lui-même analytique (utiliser la continuité de l'application "intersection" et la compacité du graphe de la relation "inclusion"); donc $\mathscr{U}_{D}$ est analytique. 
$(2) \Rightarrow(3)$. Le sous-ensemble dénombrable $D$ de $K$ étant dense dans $K$, l'espace $\mathscr{C}(K)$ muni de la topologie de la convergence simple sur $D$-que nous noterons désormais $\mathscr{C}_{D}(K)$-s'identifie au sous-ensemble de $R^{D}$ formé des fonctions uniformément continues pour $\mathscr{U}_{D}$. Le Lemme 2 termine la démonstration.

$(3) \Rightarrow(1)$. Soit $D$ une partie dénombrable dense dans $K$. L'espace $\mathscr{C}_{D}(K)$ est analytique. On pose $\mathscr{C}_{D}(K)=A$. Soit $\mathscr{F}(A ; R)$ l'ensemble des application de $A$ dans $R$.

On considère

$$
\begin{aligned}
\varphi: K & \longrightarrow \mathscr{F}(A ; R) \\
x & \longmapsto \varepsilon_{x}: f \longmapsto f(x)
\end{aligned}
$$

l'application $\varphi$ est une injection, continue lorsqu'on munit $\mathscr{F}(A ; R)$ de la topologie de la convergence simple sur $A$. L'ensemble $\varphi(K)$ est donc un compact, isomorphe à $K$, de fonctions sur l'espace $A$. Si $x \in D$, la fonction $\varphi(x)$ est continue, d'après la définition de $D$. Montrons que pour tout $x \in K$, la fonction $\varphi(x)$ est borélienne.

Soit en effet $D^{\prime}=D \cup\{x\}$. Par hypothèse, l'espace $\mathscr{C}_{D}^{\prime}(K)$ est analytique. Ecrivons $D=\left\{x_{n}\right\}$. Soit $\mathscr{F}$ la trace du filtre des voisinages de $x$ sur $D$. On a

$$
\begin{aligned}
F \in \mathscr{P}(D) ; F \in \mathscr{F} \rightleftarrows & f \in \mathscr{C}_{D}^{\prime}(K) \text { telle que } f(x)=0 \text {, et } \\
& f\left(x_{n}\right)>1 \quad \forall n \in C F .
\end{aligned}
$$

Soit $B=\left\{f \in \mathscr{C}_{D}^{\prime}(K) \mid f(x)=0\right\}$. L'ensemble $B$, fermé dans un analytique, est analytique.

Soit $\alpha$ l'application

$$
\begin{aligned}
\alpha: B & \longrightarrow \mathscr{P}(D) \\
f & \longmapsto\left\{x_{n} \in D \mid f\left(x_{n}\right)>1\right\} .
\end{aligned}
$$

On vérifie aisément que l'application $\alpha$ est borélienne; donc $\alpha(B)$ est analytique. Or le filtre $\mathscr{F}$ est l'image de $\alpha(B)$ par l'application $X \rightarrow C X$, qui est un homéomorphisme de $\mathscr{P}(D)$; le filtre $\mathscr{F}$ est donc analytique. On peut écrire $\varphi(x)=\lim _{\mathscr{\sigma}} \varphi\left(x_{n}\right)$, et déduire du Lemme 3 que $\varphi(x)$ est une fonction borélienne.

$\varphi(K)$ apparaît à présent comme un compact de fonctions boréliennes sur $A$, contenant une partie dense de fonctions continues; on sait qu'alors (voir [3]), $\varphi(K)$ est formé de fonctions de $1^{\text {ere }}$ classe sur $A$.

Soit enfin $h$ une surjection continue de $N^{N}$ sur $A$. Soit $K_{0}=$ $\{f \circ h \mid f \in \varphi(K)\}$. L'espace $K_{0}$ est un compact de Rosenthal isomorphe à $K$.

REMARQUE. Les équivalences ci-dessus montrent que les compacts 
séparables qui apparaissent "naturellement" -c'est-à-dire sans l'usage de l'axiome du choix- sont "en général" des compacts de Rosenthal; en effet, les sous-ensembles explicites des compacts métrisables sont "en général" analytiques, et même boréliens. Avec les notations ci-dessus, le filtre $\mathscr{C}_{D}$ des entourages de $\mathscr{C}$ sur $D$ sera donc une partie analytique de $\mathscr{P}(D \times D)$ si $K$ est donné explicitement. Les compacts de Rosenthal ayant de bonnes propriétés (ils sont en particulier angéliques, voir [3], Déf. 3, A) on obtient un résultat positif sur les compacts séparables "naturels".

Passons à présent aux applications. Le classe des compacts de Rosenthal est clairement stable par sous-espace fermé et par produit fini ou dénombrable. On a malheureusement le résultat négatif suivant.

Proposition 5. La classe des compacts de Rosenthal séparables n'est pas stable par image continue.

Demonstration. On va montrer qu'il existe une image continue du compact "intervalle éclaté"-c'est-à-dire $[0,1] \times\{0,1\}$ muni de l'order lexicographique- qui n'est pas un compact de Rosenthal.

Soit $X$ un sous-ensemble non analytique de $[0,1]$. Soit $K$ le compact obtenu à partir de $[0,1] \times\{0,1\}$ en identifiant let points $(x, 0)$ et $(x, 1)$ pour tout $x \in[0,1] \backslash X$; le compact $K$ est image continue de l'intervalle éclaté de façon naturelle. On définit le sous-ensemble $Y$ de $\mathscr{C}(K)$ de la façon suivante:

$$
Y=\left\{\chi_{[(x, 0),(1,1)]} \mid x \in X\right\} \cup\left\{\chi_{[(x, 1),(1,1)]} \mid x \in X\right\}
$$

si $D$ est une partie dénombrable dense de $K$, l'ensemble $Y$ apparaît comme un fermé de $\mathscr{C}_{D}(K)$; c'est en effet l'ensemble des fonctions croissantes à valeurs dans $\{0,1\}$. Si $K$ était un compact de Rosenthal, l'ensemble $Y$ serait donc analytique. Mais 1'espace $X$ est image continue de $Y$, et n'est pas analytique, ce qui est absurde.

REMARques. On peut également remarquer que l'ensemble des compacts de Rosenthal séparables a la puissance du continu, puisqu'il est image surjective de l'ensemble des parties dénombrables de l'ensemble $B_{1}\left(N^{N}\right)$, qui a la puissance du continu; or, l'intervalle éclaté a $2^{c}$ images continues non homéomorphes deux à deux.

Nous allons voir dans la suite que sous certaines conditions restrictives, on a des résultats de stabilité.

On a en effet le résultat suivant.

Proposition 6. Soit $K$ un compact de Rosenthal séparable, $\varnothing$ 
une surjection continue ouverte sur un compact $K^{\prime}$. Alors $K^{\prime}$ est un compact de Rosenthal.

Demonstration. On sait ([2]) que si $K$ est un compact de Rosenthal, $K$ contient une partie dense de points $\mathscr{G}_{j}$.

Soit donc $K$ un compact de Rosenthal séparable, $A$ l'ensemble des points $\mathscr{G}_{j}$ de $K$. Tout élément de $K$ est limite d'une suite d'éléments de $A$, puisque $K$ est angélique ([3]). On peut donc trouver une partie dénombrable dense $D$ de $K$ formée de points $\mathscr{G}_{j}$.

Soit $\varphi$ une surjection continue ouverte de $K$ sur un compact $K^{\prime}$. Soit $D^{\prime}=\varphi(D) ; D^{\prime}$ est une partie dénombrable dense de $K^{\prime}$, formée également de points $\mathscr{G}_{j}$. On considère l'application

$$
\begin{aligned}
\varphi \times \varphi: K \times K & \longrightarrow K^{\prime} \times K^{\prime} \\
(a, b) & \longmapsto(\varphi(a), \varphi(b)) .
\end{aligned}
$$

C'est clairement une application ouverte de $K \times K$ dans $K^{\prime} \times K^{\prime}$. Soit $\psi$ l'application de $P(D \times D)$ dans $P\left(D^{\prime} \times D^{\prime}\right)$ définie de la façon suivante

$$
\begin{aligned}
\psi^{\prime}=\mathscr{P}(D \times D) & \longrightarrow \mathscr{P}\left(D^{\prime} \times D^{\prime}\right) \\
X & \longmapsto \varphi \times \varphi(\bar{X}) \cap\left(D^{\prime} \times D^{\prime}\right)
\end{aligned}
$$

où $\bar{X}$ désigne l'adhérence de $X$ dans $K \times K$.

Montrons que l'application $\psi$ est borélienne. Soit $a$ et $b$ deux points de $D^{\prime} \times D^{\prime}$, et soit $\omega=\left\{Y \in P\left(D^{\prime} \times D^{\prime}\right) \mid Y \ni a, Y \nexists b\right\}$. Il suffit de montrer que pour tout ouvert $\omega$ ainsi défini, $\psi^{-1}(\omega)$ est une partie borélienne de $\mathscr{P}(D \times D)$. On a

$$
X \in \psi^{-1}(\omega) \Longleftrightarrow\left\{\begin{array}{l}
\bar{X} \cap(\varphi \times \varphi)^{-1}(a) \neq \varnothing \\
\bar{X} \cap(\varphi \times \varphi)^{-1}(b)=\varnothing .
\end{array}\right.
$$

Le point a étant un point $\mathscr{G}_{j}$, le fermé $(\varphi \times \varphi)^{-1}(a)$ est également un fermé $\mathscr{G}_{j}$. Soit $(\varphi \times \varphi)^{-1}(\alpha)=\bigcap_{n \in N} O_{n}$. On a

$$
\begin{aligned}
& \bar{X} \cap(\varphi \times \varphi)^{-1}(a) \neq \varnothing \\
& \Longleftrightarrow \forall_{n}, \bar{X} \cap O_{n} \neq \varnothing \\
& \Longleftrightarrow \forall n, X \cap O_{n} \neq \varnothing .
\end{aligned}
$$

Or, l'ensemble $\omega_{1}^{n}=\left\{X \in P(D \times D) \mid X \cap O_{n} \neq \varnothing\right\}$ est un ouvert de $P(D \times D)$, et donc $G=\left\{X \in P(D \times D) \mid \bar{X} \cap(\varphi \times \varphi)^{-1}(a) \neq \varnothing\right\}$ est un ensemble $\mathscr{G}_{\delta}$ de $P(D \times D)$.

De même on a

$$
\begin{aligned}
& \bar{X} \cap(\varphi \times \varphi)^{-1}(b)=\varnothing \\
& \Longleftrightarrow \exists V_{n} \text { tel que } \bar{X} \cap V_{n}=\varnothing, \text { où }(\varphi \times \varphi)^{-1}(b)=\bigcap_{N} V_{n} \\
& \Longleftrightarrow \exists V_{n} \text { tel que } X \cap V_{n}=\varnothing .
\end{aligned}
$$


Donc l'ensemble $G^{\prime}=\left\{X \in P(D \times D) \mid \bar{X} \cap(\varphi \times \varphi)^{-1}(b)=\varnothing\right\}$ est un ensemble $F_{\sigma}$. Or, on a $\psi^{-1}(\omega)=G \cap G^{\prime}$, ce qui montre que $\psi$ est borélienne.

Notons maintenant que le filtre des entourages de la structure uniforme de $K$ est le filtre des voisinages de la diagonale $\triangle$ de $K \times K$ dans $K \times K$. On en déduit, avec les notations du Théorème 4, que $\psi\left(\mathscr{U}_{D}\right)=\mathscr{U}_{D^{\prime}}$. En effet, l'application $\varphi$ étant continue et ouverte, l'image de tout entourage est un entourage. D'après le Théorème 4 , le filtre $\mathscr{C}_{D}$ est analytique, l'application $\psi$ étant borélienne, le filtre $\mathscr{U}_{D}^{\prime}$ est analytique.

En reprenant la méthode employée dans la démonstration de (3) $\Rightarrow(1)$ du Théorème 4 , on fait alors apparaître $K^{\prime}$ comme un compact de fonctions sur $N^{N}$ qui contient une partie dense de fonctions continues, et qui est par ailleurs séquentiellement compact, comme image continue d'un compact séquentiellement compact. On sait qu'alors ([3]), $K^{\prime}$ est formé de fonctions de $1^{\text {ere }}$ classe sur $N^{N}$; donc $K^{\prime}$ est un compact de Rosenthal.

Etudions maintenant l'espace des mesures de probabilité sur $K$.

Proposition 7. Soit $K$ un compact de Rosenthal. L'espace $\mathscr{L}_{1}^{+}(K)$ des mesures de probabilité sur $K$, muni de la topologie vague, est un compact de Rosenthal.

Demonstration. Plaçons nous tout d'abord dans le cas où $K$ est séparable. Soit $D$ une partie dénombrable dense de $K$. L'espace $\mathscr{C}_{D}(K)$ est analytique; la boule unité de $\mathscr{C}_{D}(K)$, qu'on note $B$, est fermée dans cet analytique, donc analytique. $K$ apparaît comme un compact uniformément borné de fonctions de première classe sur $B$. On sait qu'alors ([3], Théorème 5.E), l'enveloppe convexe fermée-pour la topologie de la convergence ponctuelle- de $K$ dans $\mathscr{F}(B ; R)$ est un compact de Rosenthal. Or, cette enveloppe convexe fermée s'identifie à $\mathscr{K}_{1}^{+}(K)$.

Une méthode un peu différente (dûe à $M$. Talagrand, qui nous a gentiment autorisé à la reproduire ici) permet de se passer de l'hypothèse de séparabilité.

On considère $K$ comme un compact de $B_{1}(X)$, où l'espace $X$ est polonais. Soit $Y$ l'espace somme directe des espaces $\left(X^{n}\right)_{n \geqq 1}$. L'espace $Y$ est analytique. Pour tout $\left(t_{1}, t_{2}, \cdots, t_{n}\right) \in Y$, on définit

$$
\begin{aligned}
\varphi_{t_{1}, t_{2}, \cdots, t_{n}}: K & \longrightarrow R \\
x & \longmapsto \prod_{i=1}^{n} x\left(t_{i}\right) .
\end{aligned}
$$

Les fonctions $\left(\varphi_{t}\right)_{t \in X}$ séparent les points de $K$. D'après le théo- 
rème de Stone-Weierstrass, l'espace vectoriel engendré par les $\left(\varphi_{t_{1}, t_{2}, \cdots, t_{n}}\right)_{t_{1}, t_{2}, \cdots, t_{n} \in Y}$ est normiquement dense dans $\mathscr{C}(K)$.

Les fonctions

$$
\begin{aligned}
& \tilde{x}: Y \longrightarrow R \\
& \left(t_{1}, t_{2}, \cdots t_{n}\right) \longmapsto \varphi_{t_{1}, t_{2}, \cdots, t_{n}}(x)
\end{aligned}
$$

où $x \in K$, forment un compact de fonctions de $1^{\text {ere }}$ classe sur $Y$, donc l'enveloppe convexe fermée s'identifie à $\mathscr{l}_{1}^{+}(K)$, d'où le résultat.

On en déduit la

Proposition 8. Soit $K$ un compact de Rosenthal, et $\mu$ une mesure de Radon sur $K$. Alors le support de e est séparable.

Demonstration. On peut évidemment prendre pour $\mu$ une mesure de probabilité. On sait que $\mu$ appartient à l'adhérence vague de l'ensemble des mesures de probabilité à support fini inclus dans $\operatorname{Supp}(\mu)$. Mais le compact $\mathscr{K}_{1}^{+}(K)$ étant un compact de Rosenthal, il est angélique; il existe donc une suite $\left(\mu_{n}\right)_{n \in N}$ de telles mesures convergeant vers $\mu$. Soit $F_{n}=\operatorname{Supp}\left(\mu_{n}\right)$. L'ensemble $D=\bigcup_{n \in N} F_{n}$ est un ensemble dénombrable dense dans $\operatorname{Supp}(\mu)$.

Remarque. La démonstration de la Proposition 7 permet de montrer que si $K$ est un compact de Rosenthal, tous les compacts de $\left(\mathscr{C}(K)^{*}, w^{*}\right)$ sont des compacts de Rosenthal.

Il existe, comme on l'a vu, des compacts de Rosenthal non séparables-par exemple le compactifié d'Alexandroff d'un ensemble discret de cardinal c-ce qui donne son intérêt à la Proposition 8.

II. Espace des fonctions continues sur un compact de Rosenthal. Soit $E$ un espace de Banach. On notera $\mathrm{Cyl}(E)$ la tribu "cylindrique" de $E$, c'est-à-dire la tribu engendrée par les formes linéaires continues sur $E$; d'après un résultat d'Edgar ([6], Théorème 2.3), la tribu $\operatorname{Cyl}(E)$ coïncide avec la tribu engendrée par les fonctions continues sur $(E, w)$. Par contre, la tribu $\operatorname{Cyl}(E)$ est en général distincte de la tribu des boréliens de la topologie faible. On montre, par exemple, que si $E$ est un Banach séparable ne contenant pas $\ell^{1}(N)$, alors la tribu $\operatorname{Cyl}(E)$ et la tribu de boréliens de la topologie faible coïncident si et seulement si $E^{\prime}$ est séparable.

On a le résultat suivant.

Theoreme 9. Soit $K$ un compact séparable. $K$ est un compact de Rosenthal si et seulement si l'espace mesurable ( $\mathscr{C}(K), \operatorname{Cyl}(\mathscr{C}(K)))$ est un espace souslinien. 
Demonstration. Supposons que $K$ soit un compact de Rosenthal. Soit $D$ une partie dénombrable dense dans $K$. L'espace $\mathscr{C}_{D}(K)$ est analytique d'après le Théorème 4 , et toute mesure de Radon sur $K$ est une fonction de $1^{\text {ere }}$ classe sur cet espace. La tribu $\mathrm{Cyl}(\mathscr{C}(K))$ s'identifie alors à la tribu induite par la topologie de $\mathscr{C}_{D}(K)$; elle est done souslinienne.

Inversement, supposons que $(\mathscr{C}(K), \operatorname{Cyl}(\mathscr{C}(K)))$ soit un espace mesurable souslinien. Considérons l'application identité de $(\mathscr{C}(K)$, $\operatorname{Cyl}(\mathscr{C}(K))$ ) dans $\mathscr{C}_{D}(K)$; c'est une application borélienne à valeurs dans un espace mesurable à base dénombrable; on en déduit que $\mathscr{C}_{D}(K)$ est un espace métrisable souslinien, donc un espace analytique (voir [4]); ceci pour toute partie dénombrable $D$ dense dans $K$. Le Théoréme 4 termine la démonstration.

Remarques. (1) La caractérisation ci-dessus ne s'étend pas aux compacts non séparables.

En effet, si $K$ est le compactifié d'Alexandroff d'un espace discret de cardinal $c$, la tribu $\operatorname{Cyl}(\mathscr{C}(K))$ n'est pas à base dénombrable, donc n'est pas souslinienne, mais $K$ est un compact de Rosenthal.

(2) Soit $\varphi$ une surjection continue d'un compact de Rosenthal séparable $K$ sur un compact $K_{0}$ qui n'est pas de Rosenthal. L'ensemble $F=\left\{f \circ \varphi \mid f \in \mathscr{C}\left(K_{0}\right)\right\}$ est un sous-ensemble faiblement fermé de $\mathscr{C}(K)$ qui n'est pas souslinien dans $(\mathscr{C}(K), \operatorname{Cyl}(\mathscr{C}(K)))$, et en particulier non borélien dans $(\mathscr{C}(K), \operatorname{Cyl}(\mathscr{C}(K)))$. On a donc ici un autre exemple d'un borélien de la topologie faible qui n'appartient pas à la tribu cylindrique. On voit par ailleurs que la mise en évidence de $\varphi$ revient à mettre en évidence un sous-ensemble non souslinien d'un espace souslinien, donc un sous-ensemble non analytique de $R$, d'où le caractére essentiellement nécessaire de l'exemple donné dans la démonstration de la Proposition 5.

(3) La classe des compacts de Rosenthal séparables n'étant pas stable par image continue, on ne peut espérer une bonne caractérisation en termes de topologie ou de tribu borélienne déduite d'une topologie sur $\mathscr{C}(K)$-voir la Remarque (2) ci-dessus-. Il semble que le Théorème 9 soit la bonne caractérisation en termes d'espace $\mathscr{C}(K)$.

(4) On montre aisément les équivalences suivantes: $K$ est un compact de Rosenthal $\Leftrightarrow$ il existe une partie $A$, totale dans $\mathscr{C}(K)$, souslinienne dans $(\mathscr{C}(K), \operatorname{Cyl}(\mathscr{C}(K))) \Leftrightarrow$ la tribu $\operatorname{Cyl}(\mathscr{C}(K))$ est raffinée par une tribu souslinienne.

Déduisons maintenant du Théorème 9 quelques résultats de 
stabilité.

Proposition 10. Soit $K$ un compact de Rosenthal séparable.

(1) Soit $\varphi: K \rightarrow K^{\prime}$ une surjection continue, soit $X=\{f \circ \varphi \mid f \in$ $\left.\mathscr{C}\left(K^{\prime}\right)\right\}$. S'il existe une injection continue de l'espace $\mathscr{C}(K)_{I_{X}}$ dans $\iota^{\infty}(N)$-en particulier si $\mathscr{C}(K)_{/ X}$ est séparable-le compact $K^{\prime}$ est un compact de Rosenthal.

(2) Soit $K$ un compact de Rosenthal séparable, $K^{\prime}$ un compact séparable. Si les espaces $\left(\mathscr{C}(K)\right.$, w) et $\left(\mathscr{C}\left(K^{\prime}\right)\right.$, w) sont homéomorphes, alors $K^{\prime}$ est un compact de Rosenthal.

Demonstration. (1) Soit $E$ un Banach. On a l'équivalence: il existe une injection continue de $E$ dans $\ell^{\infty}(N) \Leftrightarrow$ l'espace $\left(E^{*}\right.$, w $\left.^{*}\right)$ est séparable.

Si donc l'espace $\left(\left(\mathscr{C}(K)_{/ X}\right)^{*} w^{*}\right)$ est séparable, l'espace $\left(X^{\perp}, w^{*}\right)$ qui s'y identifie est séparable. L'espace $X$ est donc une intersection dénombrable d'hyperplans fermés de $\mathscr{C}(K)$, et donc $X \in \operatorname{Cyl}(\mathscr{C}(K))$, l'espace $\mathscr{C}\left(K^{\prime}\right)$, isomorphe à $X$, vérifie done $\left(\mathscr{C}\left(K^{\prime}\right), \operatorname{Cyl}\left(\mathscr{C}\left(K^{\prime}\right)\right)\right.$ ) souslinien; ce qui montre que $K^{\prime}$ est un compact de Rosenthal.

(2) Si les espaces $\left(\mathscr{C}(K)\right.$, w) et $\left(\mathscr{C}\left(K^{\prime}\right), w\right)$ sont homéomorphes, les espaces mesurables $(\mathscr{C}(K), \operatorname{Cyl}(\mathscr{C}(K)))$ et $\left(\mathscr{C}\left(K^{\prime}\right), \operatorname{Cyl}\left(\mathscr{C}\left(K^{\prime}\right)\right)\right)$ sont isomorphes, puisque la tribu cylindrique est la tribu engendrée par les fonctions continues; le Théorème 9 termine la démonstration.

Remarque. La démonstration du (1) ci-dessus montre que le compact des mesures de Radon de masse inférieure ou égale à 1 sur l'intervalle éclaté n'est pas héréditairement séparable; en effet, si $K^{\prime}$ est image continue de l'intervalle éclaté par $\varphi$, l'ensemble $\{f \circ \phi \mid f \in$ $\left.\mathscr{C}\left(K^{\prime}\right)\right\}^{\perp}$ n'est pas $\varkappa^{*}$-séparable. L'intervalle éclaté est donc un compact héréditairement séparable, tel que le compact des mesures de masse inférieure ou égale à 1 ne soit pas héréditairement séparable. Notons cependant qu'on montre que l'espace des mesures de probabilité sur l'intervalle éclaté est héréditairement séparable.

On a également.

Proposition 11. Soit $K$ un compact de Rosenthal, $K^{\prime}$ un espace compact. S'il existe une surjection linéaire continue de $\mathscr{C}(K)$ sur $\mathscr{C}\left(K^{\prime}\right)$, alors $K^{\prime}$ est un compact de Rosenthal.

Demonstration. Soit $\psi$ une surjection continue de $\mathscr{C}(K)$ sur $\mathscr{C}\left(K^{\prime}\right)$. Soit $K_{0}={ }^{t}{ }^{\prime}\left(\left\{\varepsilon_{x} \mid x \in K^{\prime}\right\}\right)$. L'espace $K_{0}$ est un compact homéomorphe à $K^{\prime}$, lorsqu'il est muni de la topologie * $^{*}$. D'après la Proposition 7, et la remarque qui suit la Proposition 8 , le compact $K_{0}$ est un compact de Rosenthal, ainsi que $K^{\prime}$. 
H. H. Corson a défini dans [5] la propriété ( $\mathscr{C}$ ) des espaces de Banach de la façon suivante: on dira qu'un espace de Banach $E$ a la propriété $(\mathscr{C})$ si toute famile de sous-ensembles fermés convexes de $E$ d'intersection vide contient une sous-famille dénombrable d'intersection vide. Tout espace de Banach séparable a clairement la propriété $(\mathscr{C})$. La propriété $(\mathscr{C})$ a été étudiée par $R$. Pol dans [9], qui a montré en particulier que l'espace des fonctions continues sur l'intervalle éclaté a la propriété $(\mathscr{C})$, et qui a posé la question suivante: l'espace des fonctions continues sur le "compact de Helly" $a$-t'il la propriété $(\mathscr{C})$ ? On va répondre positivement à cette question, en montrant que la propriété est vérifiée pour tout compact de Rosenthal.

Proposition 12. Soit $E$ un espace de Banach. Si l'espace mesurable $(E, \mathrm{Cyl}(E))$ est souslinien, et si le compact $\left(B_{1}\left(E^{*}\right), w^{*}\right)$ est séparable, $E$ a la propriété (C) .

Demonstration. En effet sous les hypothèses ci-dessus, le compact $\left(B_{1}\left(E^{*}\right), w^{*}\right)$ apparaît comme un compact séparable de fonctions boréliennes sur un espace souslinien, donc de fonctions boréliennes sur $N^{N}$. Le compact $\left(B_{1}\left(E^{*}\right), w^{*}\right)$ étant séparable, il est alors homéomorphe ([3]) à un compact de Rosenthal. On sait ([9]) que la propriété (C) se caractérise de la façon suivante:

$E$ a la propriété $(\mathscr{C}) \Leftrightarrow$ la boule unité $B_{1}\left(E^{*}\right)$ de $E^{*}$ munie de «* vérifie: si $x \in \bar{A} \subseteq B_{1}\left(E^{*}\right)$, il existe un sous-ensemble dénombrable $C$ de $A$ tel que $x \in \overline{C O}^{\text {ru* }}(C)$.

Or, si $\left(B_{1}\left(E^{*}\right), w^{*}\right)$ est un compact de Rosenthal, tout $x$ adhérent à $A$ est limite d'une suite d'éléments de $A$, d'après l'angélicité, et donc la propriété ci-dessus est à fortiori vérifiée.

Remarque. On voit facilement que le compact $\left(B_{1}\left(E^{*}\right)\right.$, w*) est séparable si et seulement si $E$ est isométrique à un sous-espace de $\ell^{\infty}(N)$.

On déduit de la Proposition 12 le

TheOREMe 13. Soit $K$ un compact de Rosenthal. Alors l'espace $\mathscr{C}(K)$ a la propriété (C) .

Demonstration. Si $K$ est un compact de Rosenthal séparable, l'espace $\mathscr{C}(K)$ vérifie, d'après le Théorème 9 , les hypothèses de la Proposition 12; d'où le résultat. Dans le cas général, on remarque que $\left(B_{1}\left(E^{*}\right), w^{*}\right)$, compact de Rosenthal, est angelique, d'où le resultat. 
Terminons ce travail par quelques applications des résultats de [3], en particulier du Théorème 2.F. Ces résultats se déduisant aisément de [3], sont donnés ici sans démonstration.

Sous-algebres de $\ell^{\infty}(N)$. Soit $E$ ne sous-algèbre unitaire fermée de $\ell^{\infty}(N)$, analytique dans $R^{N}$ muni de la topologie produit. Deux cas sont possibles, que nous désignerons par "type I" et "type II".

Type I. $(E, \operatorname{Cyl}(E))$ est un espace souslinien.

$\Leftrightarrow(E, \operatorname{Cyl}(E))$ est à base dénombrable.

$\Leftrightarrow\left(B_{1}\left(E^{*}\right)\right.$, w $\left.^{*}\right)$ est un compact de Rosenthal.

$\Leftrightarrow$ Pour tout application $\psi: \ell^{\infty}(N) \rightarrow \ell^{\infty}(N)$ linéaire continue, $\psi(E)$ est une partie analytique de $R^{N}$.

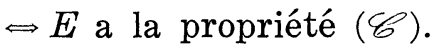

$\Leftrightarrow C$ ard $E^{*}=C$.

Type II. $\exists I \in P_{\infty}(N)$ tel que $\Pi_{I}(E)=\ell^{\infty}(I)$.

$\Leftrightarrow \exists$ une application linéaire continue $\varphi: \ell^{\infty}(N) \rightarrow E$ qui n'est pas faiblement compacte.

$\Leftrightarrow \exists$ une surjection linéaire continue $\psi: E \rightarrow \ell^{\infty}(N)$.

$\Leftrightarrow C \operatorname{ard} E^{\prime}=2^{C}$.

$\Leftrightarrow(E, \operatorname{Cyl}(E))$ est un espace mesurable qui n'est pas à base dénombrable.

On a ainsi une classification des sous-algèbres "analytiques" de $\ell^{\infty}(N)$-en particulier de celles qui sont des parties boréliennes de $R_{N^{-}}$.

Sous-algebres de Boole de $\mathscr{P}(N)$. D'après le théorème de représentation de Stone, toute sous-algèbre de Boole $A$ de $\mathscr{P}(N)$ est isomorphe à l'algèbre des ouverts-fermés d'un compact totalement discontinu $K_{A}$ séparable, et inversement. On voit aisément, en employant le Théorème 4, que si $K_{A}$ est un compact de Rosenthal, l'algèbre $A$ est une sous-algèbre analytique de $\mathscr{P}(N)$.

Inversement, soit $A$ une sous-algèbre de Boole analytique de $\mathscr{P}(N)$. Deux cas sont possibles, que nous désignerons là encore par “Type I" et “Type II", ces deux cas s'excluant mutuellement.

Pour tout $Y \in P(N)$, on note $A_{Y}=\{X \cap Y \mid X \in A\}$. L'ensemble $A_{Y}$ est une sous-algèbre de Boole de $\mathscr{P}(Y)$.

Type I. Il existe une partie infinie $Y$ de $N$ telle que $A_{Y}=\mathscr{P}(Y)$.

Type II. Pour toute partie infinie $Y$ de $N$, il existe $Z$, partie infinie de $Y$, telle que $A_{Z}$ soit l'algèbre des sous-ensembles finis ou 
cofinis de $Z$.

Remarque. On notera -sans surprise- l'aspect "Propriété de Ramsey" de ces dernières propriétés.

\section{BIBLIOGRAPHIE}

1. N. Bourbaki, Topologie générale.

2. J. Bourgain, Some remarks on compact sets of first Baire class, preprint.

3. J. Bourgain, D. H. Fremlin and M. Talagrand, Pointwise compact sets of Bairemeasurable functions, Amer. J. Math., $100 \mathrm{~N}^{\circ} 4$ (1978), 845-886.

4. J. P. R. Christensen, Topology and Borel structure, North Holland Mathematics studies, (1974).

5. H. H. Corson, The weak topology of a Banach space, Trans. Amer. Math. Soc., 101 (1961), 1-15.

6. G. A. Edgar, Measurability in Banach spaces, Indiana University J. Math., $26 \mathrm{~N}^{\circ} 4$ (1977), 663-680.

7. R. C. James, A separable somewhat reflexive Banach space with nonseparable dual, Bull. Amer. Math. Soc., 80 (1974), 738-743.

8. E. Odell and H. P. Rosenthal, A double dual characterization of separable Banach spaces containing $l^{1}$, Israel J. Math., 20 (1975), 375-384.

9. R. Pol, On a question of $H$. H. Corson and some related problems, preprints, (Warszgawa).

10. H. P. Posenthal, A characterization of Banach spaces containing $l^{1}$, Proc. Nat. Acad. Sci. U.S.A., 71 (1974), 2411-2413.

Received March 3, 1978 and in revised form January 30, 1980.

UNIVERSITE PARIS VI

4 Place Jussieu

75230 PARIS CEDEX 05

$F_{\text {RANCE }}$ 


\section{PACIFIC JOURNAL OF MATHEMATICS}

\section{EDITORS}

DONALD BABBITT (Managing Editor)

University of California

Los Angeles, CA 90024

Hugo RossI

University of Utah

Salt Lake City, UT 84112

C. C. MOORE and ANDREW OGG

University of California

Berkeley, CA 94720

\section{J. DugundjI}

Department of Mathematics

University of Southern California

Los Angeles, CA 90007

R. FinN and J. Milgram

Stanford University

Stanford, CA 94305

\section{ASSOCIATE EDITORS}
E. F. BeCKENBACH
B. H. Neumann
F. WoLF
K. YoSHIDA

\section{SUPPORTING INSTITUTIONS}

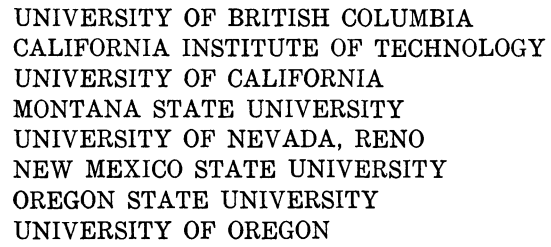

UNIVERSITY OF BRITISH COLUMBIA CALIFORNIA INSTITUTE OF TECHNOLOGY UNIVERSITY OF CALIFORNIA MONTANA STATE UNIVERSITY UNIVERSITY OF NEVADA, RENO NEW MEXICO STATE UNIVERSITY OREGON STATE UNIVERSITY UNIVERSITY OF OREGON

\author{
UNIVERSITY OF SOUTHERN CALIFORNIA \\ STANFORD UNIVERSITY \\ UNIVERSITY OF HAWAII \\ UNIVERSITY OF TOKYO \\ UNIVERSITY OF UTAH \\ WASHINGTON STATE UNIVERSITY \\ UNIVERSITY OF WASHINGTON
}

The Supporting Institutions listed above contribute to the cost of publication of this Journal, but they are not owners or publishers and have no responsibility for its content or policies.

Mathematical papers intended for publication in the Pacific Journal of Mathematics should be in typed form or offset-reproduced, (not dittoed), double spaced with large margins. Please do not use built up fractions in the text of the manuscript. However, you may use them in the displayed equations. Underline Greek letters in red, German in green, and script in blue. The first paragraph or two must be capable of being used separately as a synopsis of the entire paper. Please propose a heading for the odd numbered pages of less than 35 characters. Manuscripts, in triplicate, may be sent to any one of the editors. Please classify according to the scheme of Math. Reviews, Index to Vol. 39. Supply name and address of author to whom proofs should be sent. All other communications should be addressed to the managing editor, or Elaine Barth, University of California, Los Angeles, California, 90024.

50 reprints to each author are provided free for each article, only if page charges have been substantially paid. Additional copies may be obtained at cost in multiples of 50 .

The Pacific Journal of Mathematics is issued monthly as of January 1966. Regular subscription rate: $\$ 84.00$ a year (6 Vols., 12 issues). Special rate: $\$ 42.00$ a year to individual members of supporting institutions.

Subscriptions, orders for numbers issued in the last three calendar years, and changes of address shoud be sent to Pacific Journal of Mathematics, P.O. Box 969, Carmel Valley, CA 93924, U.S.A Old back numbers obtainable from Kraus Periodicals Co., Route 100, Millwood, NY 10546.

PUBLISHED BY PACIFIC JOURNAL OF MATHEMATICS, A NON-PROFIT CORPORATION

Printed at Kokusai Bunken Insatsusha (International Academic Printing Co., Ltd.). 8-8, 3-chome, Takadanobaba, Shinjuku-ku, Tokyo 160, Japan. 


\section{Pacific Journal of Mathematics}

\section{Vol. 91, No. 2 December, 1980}

Victor P. Camillo and Julius Martin Zelmanowitz, Dimension modules ... . . 249

Yonina S. Cooper, Stable sequences in pre-abelian categories ........... 263

Chandrakant Mahadeorao Deo and H. Ship-Fah Wong, On Berry-Esseen approximation and a functional LIL for a class of dependent random fields.........................................

H. P. Dikshit and S. N. Dubey, $|C, 1|$ summability of series associated with

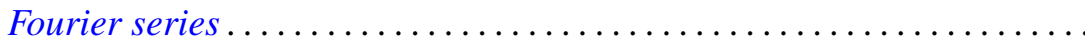

M. Edelstein, On the homomorphic and isomorphic embeddings of a semiflow into a radial flow.

Gilles Godefroy, Compacts de Rosenthal ..................... 293

James Guyker, Commuting hyponormal operators ................ 307

Thomas Eric Hall and Peter R. Jones, On the lattice of varieties of bands of

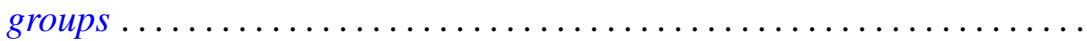

Taqdir Husain and Saleem H. Watson, Topological algebras with orthogonal Schauder bases ....................................

V. K. Jain, Some expansions involving basic hypergeometric functions of two variables. . .

Joe W. Jenkins, On group actions with nonzero fixed points ........... 363

Michael Ellsworth Mays, Groups of square-free order are scarce ........ 373

Michael John McAsey, Canonical models for invariant subspaces... 377

Peter A. McCoy, Singularities of solutions to linear second order elliptic partial differential equations with analytic coefficients by approximation methods...

Terrence Millar, Homogeneous models and decidability.

Stephen Carl Milne, A multiple series transformation of the very well poised

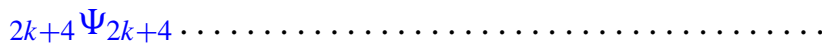

Robert Olin and James E. Thomson, Irreducible operators whose spectra are spectral sets...

Robert John Piacenza, Cohomology of diagrams and equivariant singular

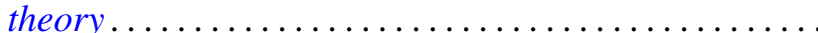

Louis Jackson Ratliff, Jr., Integrally closed ideals and asymptotic prime divisors

Robert Breckenridge Warfield, Jr., Cancellation of modules and groups and stable range of endomorphism rings.................

B. J. Day, Correction to: "Locale geometry" ...............

Stanley Stephen Page, Correction to: "Regular FPF rings" ... 